\title{
The Simulation of the Apple Tree Form's Effects on Its Photosynthetic Efficiency
}

\author{
Lin Hu, Guomin Zhou, and Yun Qiu \\ Agricultural Information Institute of CAAS, Beijing, P.R. China
}

\begin{abstract}
The form of the Apple tree is decided by the organ position of the tree, it affects not ONLY the relationship between the vegetative growth and the fruit production, but also the fruit quality. In this research, the continuous growth for 3 years was gotten with polhemus fastrak, and the apple tree model was built in the POV-ray to simulate the interception of the sunlight. A mathematic model was built between the form and the photosynthetic efficiency, the growth as the index of the form while the interception of the light as the index of the photosynthetic efficiency. Utilizing this model to analyses the effects of the form on the apple tree's photosynthetic efficiency, this result show a reference for the apple trees management.
\end{abstract}

Keywords: apple trees, light, intercept ratio, mathematic model, fine management, simulate.

\section{Introduction}

The study of plant architecture emerged as a new scientific discipline some 30 years ago. Plant architecture is a term applied to the organization of plant components in space which can change with time (2007, Daniel Barthelemy). Plant architecture is introduced into the research of the apple tree (2003, E. Costes). One of the researches is about the interactions between light and vegetation (2005, Rodrigo A.). The geometrical structure of the vegetation canopy, i.e. the location, shape and orientation of the apple tree elements determine the light distribution in the canopy (2005, J. Phattaralerphong). Many methods for the measurement of canopy geometry may involve direct measurement or may be inferred from radiation measurement using light sensor. Of the direct measurements, current induction in magnetic fields is the most convenient one.

In this paper, we use a polheumes fastrak to measure the parameters of the apple tree structure, such as the node, internodes, orientation angle, etc. the measured data was used to descript the apple tree in POV-Ray (Persistence of Vision Ray-Tracer). POV-Ray is a high-quality, freely available ray-tracing software package that is available for PC, Macintosh and UNIX platforms. POV-Ray is used in every industry widely, such as chemistry, art painting, architecture, agriculture, remote sensing and medicine. We simulate the light transfer in the crown, and evaluate the affection of the apple tree architecture on the interception of the sunlight. 


\section{Materials and Methods}

The test area is located at the Fruit Research Institute of Chinese Academy of Agricultural sciences (Figure 1).

The apple tree is HuaHong, a variety of Fuji apple, bred by the institute own self. We began this project form 2006 , for about 4 years.

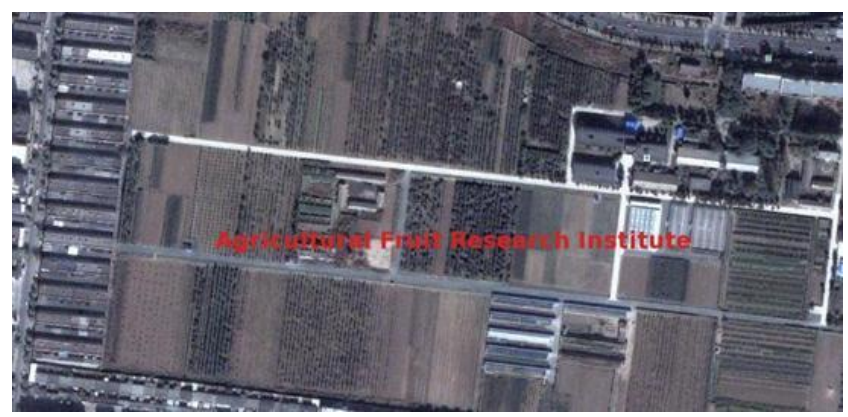

Fig. 1. Agricultural Fruit Research Institute

The data of the apple tree was measured with polhemus fastrak (1988, $\mathrm{H}$. Sinoquet), the error is $1 \mathrm{~mm}$ per meter in theory, but it could be about $1 \mathrm{~cm}$ outside for the wind and other elements.

\subsection{Topology and Geometry}

The apple tree's structure is descripting as module which can be divided in detail into two components, i.e. stem and bud. A bud develops into a flower or a branch.

We define the Apple tree structure with key points which can be divided into two kinds. One is the form key point and the other is the functional key point.

The form key point (Figure 2) is such a point which decide the apple tree form, if use a single sentence to descript, that is such a point it is not in the straight branch, or branch direction is horizontal or vertical (1999, C. Godin).

These import points can be measured by polhemus fastrak.

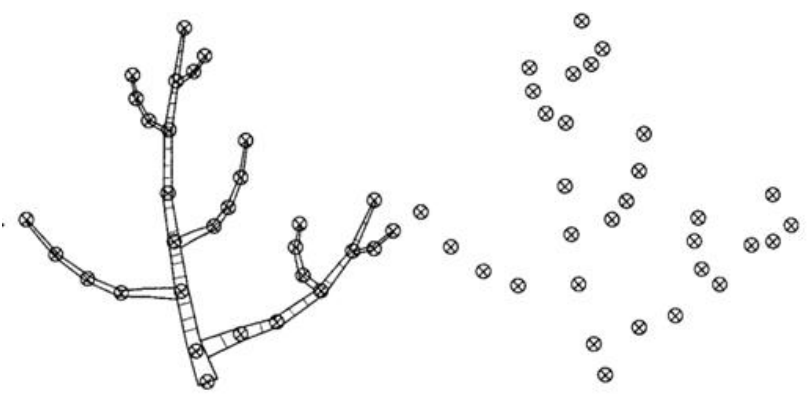

Fig. 2. The form key points 


\subsection{Data Structure}

The point data is descript with five items, i.e. point type, point-function, pre-point, next-point and the current point orientation $(\mathrm{x}, \mathrm{y}, \mathrm{z}, \mathrm{r})$, as table 1.

Table 1. The description of a point

\begin{tabular}{|c|c|c|c|c|}
\hline type & func & Pre & Next & orient \\
\hline fruit & Func & & & $(\mathrm{x}, \mathrm{y}, \mathrm{z}, \mathrm{r})$ \\
Leaf & form & & & \\
$\begin{array}{c}\text { Bud } \\
\text { branch }\end{array}$ & & & & \\
\hline
\end{tabular}

It is very convenient to conscript the apple tree structure in such a topology (Figure 3), and can be implemented in computer language easily.

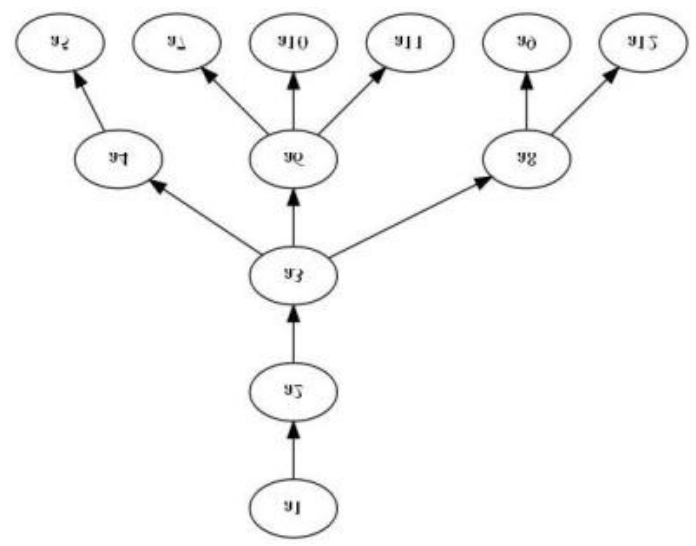

Fig. 3. Tree topology

\subsection{Apple Tree Simulate}

The apple tree data took with polhemus fastrak is stored in data file. The $3 \mathrm{~d}$ apple tree is implemented by POV-ray with the data reformatted from point data to triangle data with python.

Python is a very popular script language (2010, python development team), is used in many industry. We use python to write the measured data according to the POVray format.

POV-ray is ray trace software, a POV-ray file mainly include an object, lights and camera.

The apple tree POV-ray file includes the sun, the camera and the tree, the tree is composed by meshes (2005, Loch B.), and the mesh is compound by triangles. 
The frame of the POV-ray program is as the follow:

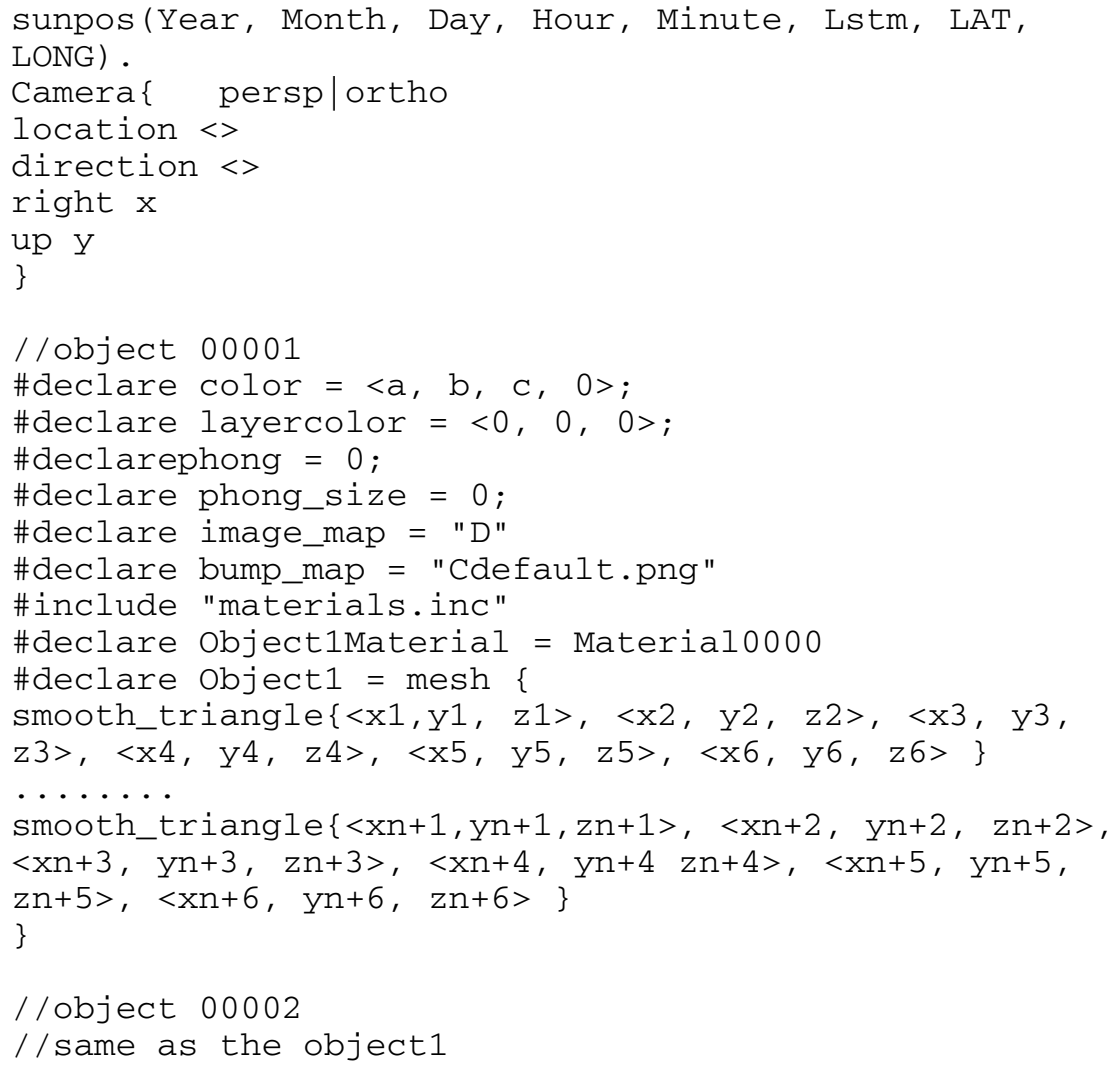

One object is an internodes, every $(\mathrm{x}, \mathrm{y}, \mathrm{z})$ is measured with polhemus fastrak.

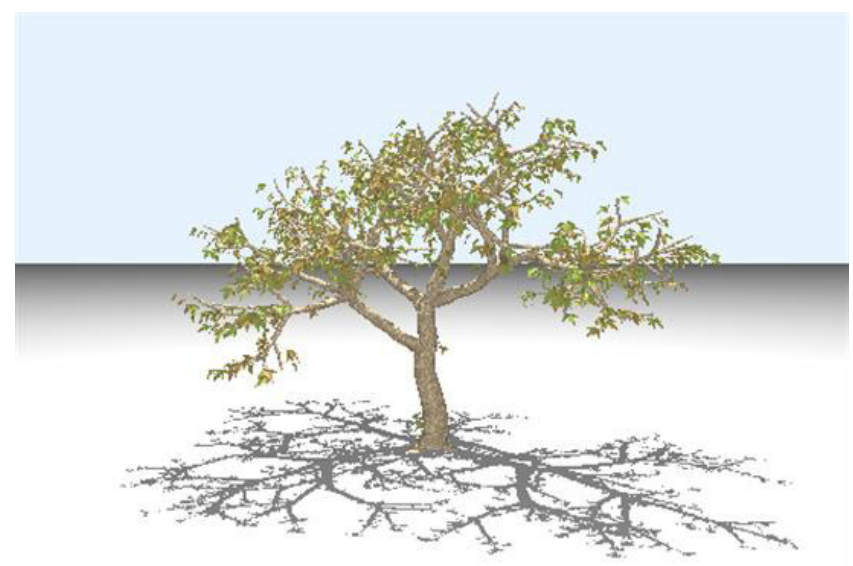

Fig. 4. Apple tree with shadow 
In order to simulate the light interception of the apple tree (2005, D. A. King), we calculate the statistical parameters based on the data measured with polhemus fastrak (2005, C. Bassette). The stem length, the probability of the branch and the leaf, the average of the radius of the stem and the branches, etc... we model a virtual apple tree using the statistic parameters (2002, D. A. Pouliot), and the demonstration picture as the figure 4 and figure 5.

In the POV-ray scene, we simulate a fisheye lens to take pictures of the apple tree's crown while we are altering the apple tree parameters (Gilles Tran), shown as the figure. 6 . The picture after segmentation is as figure 7 .

The proportion of the apple tree in the image stands for the sunlight interception, and the increment of the apple tree in the diameter for the apple tree mass increase. We develop a simulate model which present the relationship between the sunlight interception and the apple structure.

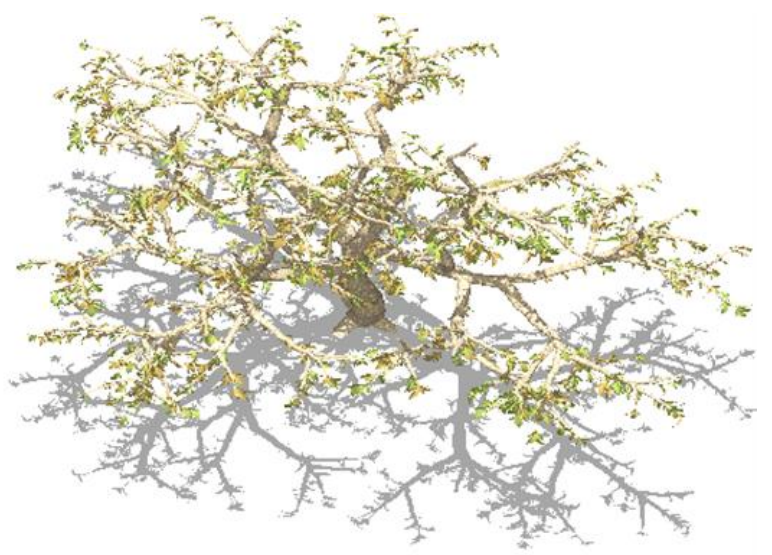

Fig. 5. Perspective of Apple tree

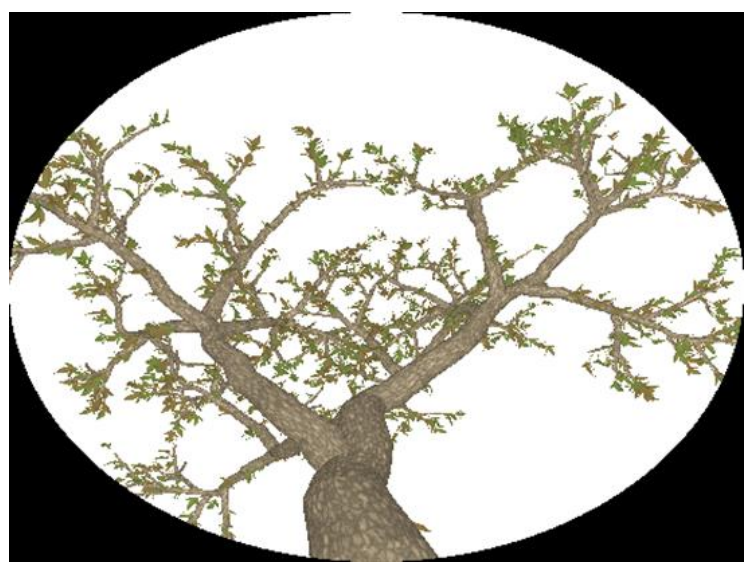

Fig. 6. The crown of the tree in fisheye 


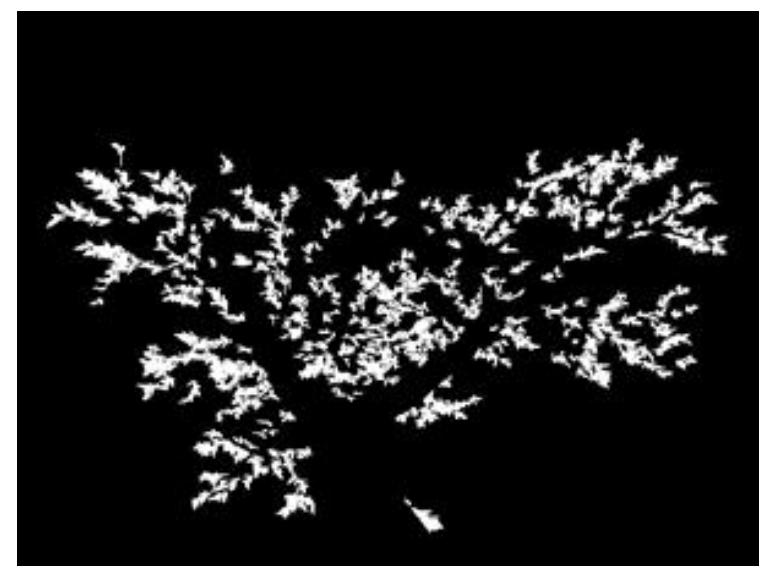

Fig. 7. The segmentation of the apple tree crown

\section{Test Results}

In this test, two factors, the branch angle and the branch radius, was tested, the branch angle was tested at level 15, 30, 45, 60 and 90 degree, and the branch radius was at $0.7 \mathrm{~cm}, 1,7 \mathrm{~cm}, 2.7 \mathrm{~cm}$. the test result is as the table 2 .

Table 2. The statics data of the segmented picture

\begin{tabular}{|c|c|c|c|c|c|}
\hline No. & Branch angel & Branch radius & Area/box & sum & stdev \\
\hline 1 & 15 & 0.7 & 0.93 & 142 & 0.16 \\
\hline 2 & 15 & 1.7 & 0.94 & 143.04 & 0.15 \\
\hline 3 & 15 & 2.7 & 0.91 & 123 & 0.18 \\
\hline 4 & 30 & 0.7 & 0.91 & 544 & 0.19 \\
\hline 5 & 30 & 1.7 & 0.91 & 501.46 & 0.2 \\
\hline 6 & 30 & 2.7 & 0.9 & 456.06 & 0.2 \\
\hline 7 & 45 & 0.7 & 0.9 & 941.05 & 0.21 \\
\hline 8 & 45 & 1.7 & 0.88 & 823 & 0.22 \\
\hline 9 & 45 & 2.7 & 0.86 & 754.38 & 0.23 \\
\hline 10 & 60 & 0.7 & 0.9 & 1234.62 & 0.21 \\
\hline 11 & 60 & 1.7 & 0.88 & 1056.71 & 0.22 \\
\hline 12 & 60 & 2.7 & 0.85 & 846.27 & 0.23 \\
\hline 13 & 90 & 0.7 & 0.9 & 1504 & 0.21 \\
\hline 14 & 90 & 1.7 & 0.89 & 1327.17 & 0.22 \\
\hline 15 & 90 & 2.7 & 0.89 & 1106.68 & 0.22 \\
\hline
\end{tabular}


The "Area/box" shows the ratio of the leaves in the whole area of the picture, and the sum means the total numbers of the pixel of the apple tree leaves.

The figure 8 shows the relationship between the interception and the branch angles, the horizontal axis shows the branch angles, and the vertical axis shows the numbers of the pixels the leaves occupied.

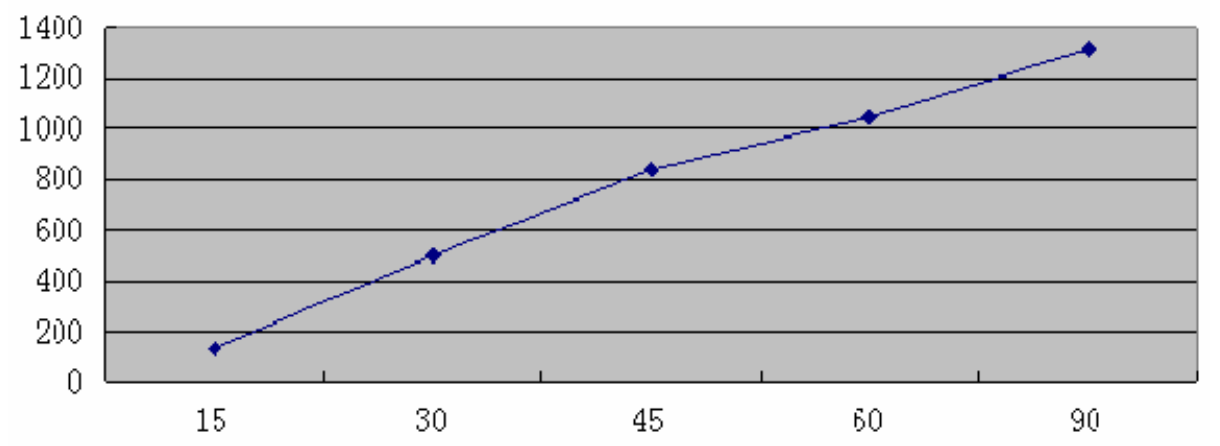

Fig. 8. The relationship between the branch angle and the light interception

The formula of the angle and the light interception is a logarithmic line, and $\mathrm{R}$ is near 1 . The relationship shows as the equation 1 .

$$
\text { Light_interception }=670.09 * \ln (\text { angle })-1713.8
$$

\section{Discuses}

The branch angle is the most important factor (1995, C. Jourdan) which affects the apple tree light interception. The result shows that the relationship between the branch angel and the light interception is a logarithmic line.

The relationship between the branch radius and the light interception cannot be described incorrectly, because when the radius of the stem increases, it will cover a big block of the branches and leaves, as shown in figure 7 . From table 2, there is an inverse ratio between the two factors. Obviously, it is contrary to the general knowledge. In future, we will find a more reasonable and fitness methods to solve this problem.

\section{References}

1. Godin, C., Costes, E., Sinoquet, H.: A Method for Describing Plant Architecture which Integrates Topology and Geometry. Annals of Botany 84, 343-357 (1999)

2. Bassette, C., Bussiére, F.: 3-D modeling of the banana architecture for simulation of rainfall interception parameters. Agricultural and Forest Meteorology 129, 95-100 (2005)

3. Jourdan, C., Rey, H., Guédon, Y.: Architectural analysis and modelling of the branching process of the young oil-palm root system. Plant and Soil 177, 63-72 (1995) 
4. Barthelemy, D., Caraglio, Y.: Plant Architecture: A Dynamic, Multilevel and Comprehensive Approach to Plant Form, Structure and Ontogeny. Annals of Botany 99, 375-407 (2007)

5. King, D.A., Davies, S.J., et al.: Tree growth is related to light interception and wood density in two mixed dipterocarp forests of Malaysia. Functional Ecology 19, 445-453 (2005)

6. Pouliot, D.A., King, D.J., Bell, F.W., Pitt, D.G.: Automated tree crown detection and delineation in high-resolution digital camera imagery of coniferous forest regeneration. Remote Sensing of Environment 82, 322-334 (2002)

7. Costes, E., Sinoquet, H., et al.: Exploring Within-tree Architectural Development of Two Apple Tree Cultivars over 6 Years. Annals of Botany 91, 91-104 (2003)

8. Gilles Tran. MakeTree v1.0., http: / /www. oyonale.com

9. Sinoquet, H., Thanisawanyangkura, S., et al.: Characterization of the light environment in canopies using 3D digitising and Image Processing. Annals of Botany 82, 203-212 (1988)

10. Phattaralerphong, J., Sinoquet, H.: A method for 3D reconstruction of tree crown volume from photographs: assessment with 3D-digitized plants. Tree Physiology 25, 1229-1242 (2005)

11. Loch, B., Belward, J., Hanan, J.: Application of surface fitting techniques for the representation of leaf surfaces. In: Zerger, A., Argent, R. (eds.) International Congress on Modelling and Simulation, pp. 1272-1278. MODSIM Press, Melbourne (2005)

12. Python development team. Python v2.6.5 document, http://www.python.org/ (last updated March 19, 2010)

13. Gutiérrez, R.A., Shasha, D.E., Coruzzi, G.M.: Systems Biology for the Virtual Plant. Plant Physiology 138, 550-554 (2005) 